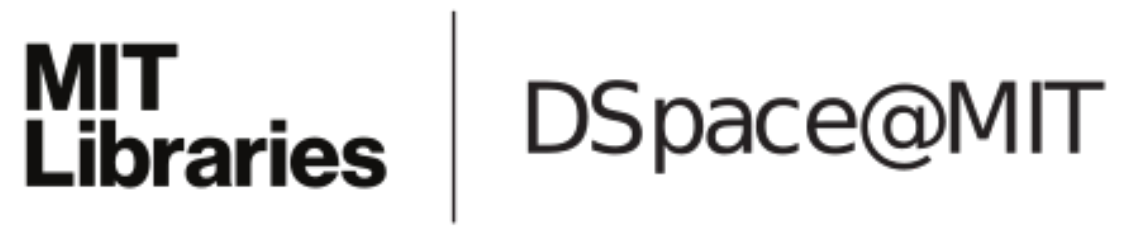

\author{
MIT Open Access Articles
}

On the Chvátal-Gomory closure of a compact convex set

The MIT Faculty has made this article openly available. Please share how this access benefits you. Your story matters.

Citation: Dadush, Daniel et al. "On the Chvátal-Gomory Closure of a Compact Convex Set." Mathematical Programming 145, 1-2 (March 2013): 327-348. doi:10.1007/s10107-013-0649-9. () 2013 The Author(s)

As Published: http://dx.doi.org/10.1007/S10107-013-0649-9

Publisher: Springer-Verlag

Persistent URL: http://hdl.handle.net/1721.1/121067

Version: Author's final manuscript: final author's manuscript post peer review, without publisher's formatting or copy editing

Terms of use: Creative Commons Attribution-Noncommercial-Share Alike 


\title{
On the Chvátal-Gomory Closure of a Compact Convex Set
}

\author{
Daniel Dadush ${ }^{1}$, Santanu S. Dey ${ }^{1}$, and Juan Pablo Vielma ${ }^{2}$ \\ ${ }^{1}$ H. Milton Stewart School of Industrial and Systems Engineering, Georgia Institute \\ of Technology, Atlanta, GA 30332, USA \\ dndadush@gatech.edu, santanu.dey@isye.gatech.edu \\ 2 Department of Industrial Engineering, University of Pittsburgh, 1048 Benedum \\ Hall, Pittsburgh, PA 15261, USA \\ jvielma@pitt.edu
}

\begin{abstract}
In this paper, we show that the Chvátal-Gomory closure of any compact convex set is a rational polytope. This resolves an open question of Schrijver [15] for irrational polytopes ${ }^{3}$, and generalizes the same result for the case of rational polytopes [15], rational ellipsoids [7] and strictly convex bodies [6].
\end{abstract}

Keywords: Chvátal-Gomory Closure, Compact Sets

\section{Introduction}

Gomory [11] introduced the Gomory fractional cuts, also known as ChvátalGomory (CG) cuts [5], to design the first finite cutting plane algorithm for Integer Linear Programming (ILP). Since then, many important classes of facetdefining inequalities for combinatorial optimization problems have been identified as CG cuts. For example, the classical Blossom inequalities for general Matching [9] - which yield the integer hull - and Comb inequalities for the Traveling Salesman problem [12,13] are both CG cuts over the base linear programming relaxations. CG cuts have also been effective from a computational perspective; see for example $[2,10]$. Although CG cuts have traditionally been defined with respect to rational polyhedra for ILP, they straightforwardly generalize to the nonlinear setting and hence can also be used for convex Integer Nonlinear Programming (INLP), i.e. the class of discrete optimization problems whose continuous relaxation is a general convex optimization problem. CG cuts for non-polyhedral sets were considered implicitly in $[5,15]$ and more explicitly in $[4,6,7]$. Let $K \subseteq \mathbb{R}^{n}$ be a closed convex set and let $h_{K}$ represent its support function, i.e. $h_{K}(a)=\sup \{\langle a, x\rangle: x \in K\}$. Given $a \in \mathbb{Z}^{n}$, we define the CG cut for $K$ derived from $a$ as the inequality

$$
\langle a, x\rangle \leq\left\lfloor h_{K}(a)\right\rfloor .
$$

${ }^{3}$ After the completion of this work, it has been brought to our notice that the polyhedrality of the Chvátal-Gomory Closure for irrational polytopes has recently been shown independently by J. Dunkel and A. S. Schulz in [8]. The proof presented in this paper has been obtained independently. 
The CG closure of $K$ is the convex set whose defining inequalities are exactly all the CG cuts for $K$. A classical result of Schrijver [15] is that the CG closure of a rational polyhedron is a rational polyhedron. Recently, we were able to verify that the CG closure of any strictly convex body ${ }^{4}$ intersected with a rational polyhedron is a rational polyhedron $[7,6]$. We remark that the proof requires techniques significantly different from those described in [15].

While the intersections of strictly convex bodies with rational polyhedra yield a large and interesting class of bodies, they do not capture many natural examples that arise in convex INLP. For example, it is not unusual for the feasible region of a semi-definite or conic-quadratic program [1] to have infinitely many faces of different dimensions, where additionally a majority of these faces cannot be isolated by intersecting the feasible region with a rational supporting hyperplane (as is the case for standard ILP with rational data). Roughly speaking, the main barrier to progress in the general setting has been a lack of understanding of how CG cuts act on irrational affine subspaces (affine subspaces whose defining equations cannot be described with rational data).

As a starting point for this study, perhaps the simplest class of bodies where current techniques break down are polytopes defined by irrational data. Schrijver considers these bodies in [15], and in a discussion section at the end of the paper, he writes ${ }^{5}$ :

"We do not know whether the analogue of Theorem 1 is true in real spaces. We were able to show only that if $P$ is a bounded polyhedron in real space, and $P^{\prime}$ has empty intersection with the boundary of $P$, then $P^{\prime}$ is a (rational) polyhedron."

In this paper, we prove that the CG closure of any compact convex set ${ }^{6}$ is a rational polytope, thus also resolving the question raised in [15]. As seen by Schrijver [15], most of the "action" in building the CG closure will indeed take place on the boundary of $K$. While the proof presented in this paper has some high level similarities to the one in [6], a substantially more careful approach was required to handle the general facial structure of a compact convex set (potentially infinitely many faces of all dimensions) and completely new ideas were needed to deal with faces having irrational affine hulls (including the whole body itself).

This paper is organized as follows. In Section 2 we introduce some notation, formally state our main result and give an overview of the proof. We then proceed with the full proof which is presented in Sections 3-5. In Section 6, we present

${ }^{4}$ A full dimensional compact convex set whose only non-trivial faces are vertices, i.e. of dimension 0 .

${ }^{5}$ Theorem 1 in [15] is the result that the CG closure is a polyhedron. $P^{\prime}$ is the notation used for CG closure in [15]

${ }^{6}$ If the convex hull of integer points in a convex set is not polyhedral, then the CG closure cannot be expected to be polyhedral. Since we do not have a good understanding of when this holds for unbounded convex sets, we restrict our attention here to the CG closure of compact convex sets. 
a generalization of Integer Farkas' Lemma that is a consequence of the proof techniques developed in this paper.

\section{Definitions, Main Result and Proof Idea}

Definition 1 (CG Closure). For a convex set $K \subseteq \mathbb{R}^{n}$ and $S \subseteq \mathbb{Z}^{n}$ let $C C(K, S):=\bigcap_{a \in S}\left\{x \in \mathbb{R}^{n}:\langle x, y\rangle \leq\left\lfloor h_{K}(y)\right\rfloor\right\}$. The $C G$ closure of $K$ is defined to be the set $C C(K):=C C\left(K, \mathbb{Z}^{n}\right)$.

The following theorem is the main result of this paper.

Theorem 1. If $K \subseteq \mathbb{R}^{n}$ is a non-empty compact convex set, then $C C(K)$ is finitely generated. That is, there exists $S \subseteq \mathbb{Z}^{n}$ such that $|S|<\infty$ and $C C(K)=$ $C C(K, S)$. In particular $C C(K)$ is a rational polyhedron.

We will use the following definitions and notation: For $x, y \in \mathbb{R}^{n}$, let $[x, y]=$ $\{\lambda x+(1-\lambda) y: 0 \leq \lambda \leq 1\}$ and $(x, y)=[x, y] \backslash\{x, y\}$. Let $B^{n}:=\left\{x \in \mathbb{R}^{n}:\right.$ $\|x\| \leq 1\}$ and $S^{n-1}:=\operatorname{bd}\left(B^{n}\right)$. (bd stands for boundary.) For a convex set $K$ and $v \in \mathbb{R}^{n}$, let $H_{v}(K):=\left\{x \in \mathbb{R}^{n}:\langle v, x\rangle \leq h_{K}(v)\right\}$ denote the supporting halfspace defined by $v$ for $K$, and let $H_{v}^{=}(K):=\left\{x \in \mathbb{R}^{n}:\langle v, x\rangle=h_{K}(v)\right\}$ denote the supporting hyperplane. $F \subseteq K$ is a face of $K$ if for every line segment $[x, y] \subseteq K,[x, y] \cap F \neq \emptyset \Rightarrow[x, y] \subseteq F$. A face $F$ of $K$ is proper if $F \neq K$. Let $F_{v}(K):=K \cap H_{v}^{=}(K)$ denote the face of $K$ exposed by $v$. If the context is clear, then we drop the $K$ and simply write $H_{v}, H_{v}^{=}$and $F_{v}$. For $A \subseteq \mathbb{R}^{n}$, let aff $(A)$ denote the smallest affine subspace containing $A$. Furthermore let $\operatorname{aff}_{I}(A):=\operatorname{aff}\left(\operatorname{aff}(A) \cap \mathbb{Z}^{n}\right)$, i.e. the largest integer subspace in $\operatorname{aff}(A)$.

We present the outline of the proof for Theorem 1 . The proof proceeds by induction on the dimension of $K$. The base case ( $K$ is a single point) is trivial. By the induction hypothesis, we can assume that $(\dagger)$ every proper exposed face of $K$ has a finitely generated CG closure. We build the CG closure of $K$ in stages, proceeding as follows:

1. (Section 3) For $F_{v}$, a proper exposed face, where $v \in \mathbb{R}^{n}$, show that $\exists S \subseteq \mathbb{Z}^{n}$, $|S|<\infty$ such that $C C(K, S) \cap H_{v}^{=}=C C\left(F_{v}\right)$ and $C C(K, S) \subseteq H_{v}$ using $(\dagger)$ and by proving the following:

(a) (Section 3.1) A CG cut for $F_{v}$ can be rotated or "lifted" to a CG cut for $K$ such that points in $F_{v} \cap \operatorname{aff}_{I}\left(H_{v}^{=}\right)$separated by the original CG cut for $F_{v}$ are separated by the new "lifted" one.

(b) (Section 3.2) A finite number of CG cuts for $K$ separate all points in $F_{v} \backslash \operatorname{aff}_{I}\left(H_{v}^{=}\right)$and all points in $\mathbb{R}^{n} \backslash H_{v}$.

2. (Section 4) Create an approximation $C C(K, S)$ of $C C(K)$ such that (i) $|S|<$ $\infty$, (ii) $C C(K, S) \subseteq K \cap \operatorname{aff}_{I}(K)$ (iii) $C C(K, S) \cap \operatorname{relbd}(K)=C C(K) \cap$ $\operatorname{relbd}(K)$. This is done in two steps:

(a) (Section 4.1) Using the lifted CG closures of $F_{v}$ from 1. and a compactness argument on the sphere, create a first approximation $C C(K, S)$ satisfying (i) and (ii). 
(b) (Section 4.2) Noting that $C C(K, S) \cap \operatorname{relbd}(K)$ is contained in the union of a finite number of proper exposed faces of $K$, add the lifted CG closures for each such face to $S$ to satisfy (iii).

3. (Section 5) We establish the final result by showing that there are only a finite number of CG cuts which separate a least one vertex of the approximation of the CG closure from (2).

\section{$3 \quad C C(K, S) \cap H_{v}^{=}=C C\left(F_{v}\right)$ and $C C(K, S) \subseteq H_{v}$}

When $K$ is a rational polyhedron, a key property of the CG closure is that for every face $F$ of $K$, we have that $(*) C C(F)=F \cap C C(K)$. In this setting, a relatively straightforward induction argument coupled with $(*)$ allows one to construct the approximation of the CG closure described above. In our setting, where $K$ is compact convex, the approach taken is similar in spirit, though we will encounter significant difficulties. First, since $K$ can have infinitely many faces, we must couple our induction with a careful compactness argument. Second and more significantly, establishing $(*)$ for compact convex sets is substantially more involved than for rational polyhedra. As we will see in the following sections, the standard lifting argument to prove $(*)$ for rational polyhedra cannot be used directly and must be replaced by a more involved two stage argument.

\subsection{Lifting CG Cuts}

To prove $C C(F)=F \cap C C(K)$ one generally uses a 'lifting approach', i.e., given a $C G$ cut $C C(F,\{w\})$ for $F, w \in \mathbb{Z}^{n}$, we show that there exists a CG cut $C C\left(K,\left\{w^{\prime}\right\}\right)$ for $K, w^{\prime} \in \mathbb{Z}^{n}$, such that

$$
C C\left(K,\left\{w^{\prime}\right\}\right) \cap \operatorname{aff}(F) \subseteq C C(F,\{w\}) \cap \operatorname{aff}(F) .
$$

To prove (2) when $K$ is a rational polyhedron, one proceeds as follows. For the face $F$ of $K$, we compute $v \in \mathbb{Z}^{n}$ such that $F_{v}(K)=F$ and $h_{K}(v) \in \mathbb{Z}$. For $w \in \mathbb{Z}^{n}$, we return the lifting $w^{\prime}=w+l v, l \in \mathbb{Z}_{>0}$, where $l$ is chosen such that $h_{K}\left(w^{\prime}\right)=h_{F}\left(w^{\prime}\right)$. For general convex bodies though, neither of these steps may be achievable. When $K$ is strictly convex however, in [6] we show that the above procedure can be generalized. First, every proper face $F$ of $K$ is an exposed vertex, hence $\exists x \in K, v \in \mathbb{R}^{n}$ such that $F=F_{v}=\{x\}$. For $w \in \mathbb{Z}^{n}$, we show that setting $w^{\prime}=w+v^{\prime}$, where $v^{\prime}$ is a fine enough Dirichlet approximation (see Theorem 2 below) to a scaling of $v$ is sufficient for (2). In the proof, we critically use that $F$ is simply a vertex. In the general setting, when $K$ is a compact convex set, we can still meaningfully lift CG cuts, but not from all faces and not with exact containment. First, we only guarantee lifting for an exposed face $F_{v}$ of $K$. Second, when lifting a CG cut for $F_{v}$ derived from $w \in \mathbb{Z}^{n}$, we only guarantee the containment on $\operatorname{aff}_{I}\left(H_{v}^{=}\right)$, i.e. $C C\left(K, w^{\prime}\right) \cap \operatorname{aff}_{I}\left(H_{v}^{=}\right) \subseteq C C(F, w) \cap \operatorname{aff}_{I}\left(H_{v}^{=}\right)$. This lifting, Proposition 1 below, uses the same Dirichlet approximation technique as in [6] but with a more careful analysis. Since we only guarantee the behavior of 
the lifting $w^{\prime}$ on $\operatorname{aff}_{I}\left(H_{v}^{=}\right)$, we will have to deal with the points in $\operatorname{aff}(F) \backslash \operatorname{aff}_{I}\left(H_{v}^{=}\right)$ separately, which we discuss in the next section.

The next lemma describes the central mechanics of the lifting process explained above. The sequence $\left(w_{i}\right)_{i=1}^{\infty}$ will eventually denote the sequence of Dirichlet approximates of the scaling of $v$ added to $w$, where one of these will serve as the lifting $w^{\prime}$. We skip the proof due to lack of space.

Lemma 1. Let $K \subseteq \mathbb{R}^{n}$ be a compact convex set. Take $v, w \in \mathbb{R}^{n}, v \neq 0$. Let $\left(w_{i}, t_{i}\right)_{i=1}^{\infty}, w_{i} \in \mathbb{R}^{n}, t_{i} \in \mathbb{R}_{+}$be a sequence such that

$$
\text { a. } \lim _{i \rightarrow \infty} t_{i}=\infty, \quad b . \lim _{i \rightarrow \infty} w_{i}-t_{i} v=w .
$$

Then for every $\epsilon>0$ there exists $N_{\epsilon} \geq 0$ such that for all $i \geq N_{\epsilon}$

$$
h_{K}\left(w_{i}\right)+\epsilon \geq t_{i} h_{K}(v)+h_{F_{v}(K)}(w) \geq h_{K}\left(w_{i}\right)-\epsilon .
$$

Theorem 2 (Dirichlet's Approximation Theorem). Let $\left(\alpha_{1}, \ldots, \alpha_{l}\right) \in \mathbb{R}^{l}$. Then for every positive integer $N$, there exists $1 \leq n \leq N$ such that $\max _{1 \leq i \leq l} \mid n \alpha_{i}-$ $\left\lfloor n \alpha_{i}\right\rceil \mid \leq 1 / N^{1 / l}$.

Proposition 1. Let $K \subseteq \mathbb{R}^{n}$ be a compact and convex set, $v \in \mathbb{R}^{n}$ and $w \in \mathbb{Z}^{n}$. Then $\exists w^{\prime} \in \mathbb{Z}^{n}$ such that $C C\left(K, w^{\prime}\right) \cap \operatorname{aff}_{I}\left(H_{v}^{=}(K)\right) \subseteq C C(K, w) \cap \operatorname{aff}_{I}\left(H_{v}^{=}(K)\right)$.

Proof. First, by possibly multiplying $v$ by a positive scalar we may assume that $h_{K}(v) \in \mathbb{Z}$. Let $S=\operatorname{aff}_{I}\left(H_{v}^{=}(K)\right)$. We may assume that $S \neq \emptyset$, since otherwise the statement is trivially true.

From Theorem 2 for any $v \in \mathbb{R}^{n}$ there exists $\left(s_{i}, t_{i}\right)_{i=1}^{\infty}, s_{i} \in \mathbb{Z}^{n}, t_{i} \in \mathbb{N}$ such that (a.) $t_{i} \rightarrow \infty$ and (b.) $\left\|s_{i}-t_{i} v\right\| \rightarrow 0$. Now define the sequence $\left(w_{i}, t_{i}\right)_{i=1}^{\infty}$, where $w_{i}=w+s_{i}, i \geq 1$. Note that the sequence $\left(w_{i}, t_{i}\right)$ satisfies (3) and hence by Lemma 1 for any $\epsilon>0$, there exists $N_{\epsilon}$ such that (4) holds. Let $\epsilon=\frac{1}{2}\left(1-\left(h_{F_{v}(K)}(w)-\left\lfloor h_{F_{v}(K)}(w)\right\rfloor\right)\right)$, and let $N_{1}=N_{\epsilon}$. Note that $\left\lfloor h_{F_{v}(K)}(w)+\right.$ $\epsilon\rfloor=\left\lfloor h_{F_{v}(K)}(w)\right\rfloor$. Hence, since $h_{K}(v) \in \mathbb{Z}$ by assumption, for all $i \geq N_{1}$ we have that $\left\lfloor h_{K}\left(w_{i}\right)\right\rfloor \leq\left\lfloor t_{i} h_{K}(v)+h_{F_{v}(K)}(w)+\epsilon\right\rfloor=t_{i} h_{K}(v)+\left\lfloor h_{F_{v}(K)}(w)+\epsilon\right\rfloor=$ $t_{i} h_{K}(v)+\left\lfloor h_{F_{v}(K)}(w)\right\rfloor$.

Now pick $z_{1}, \ldots, z_{k} \in S \cap \mathbb{Z}^{n}$ such that aff $\left(z_{1}, \ldots, z_{k}\right)=S$ and let $R=$ $\max \left\{\left\|z_{j}\right\|: 1 \leq j \leq k\right\}$. Choose $N_{2}$ such that $\left\|w_{i}-t_{i} v-w\right\| \leq \frac{1}{2 R}$ for $i \geq N_{2}$. Now note that for $i \geq N_{2},\left|\left\langle z_{j}, w_{i}\right\rangle-\left\langle z_{j}, t_{i} v+w\right\rangle\right|=\left|\left\langle z_{j}, w_{i}-t_{i} v-w\right\rangle\right| \leq$ $\left\|z_{j}\right\|\left\|w_{i}-t_{i} v-w\right\| \leq R \frac{1}{2 R}=\frac{1}{2} \quad \forall j \in\{1, \ldots, k\}$.

Next note that since $z_{j}, w_{i} \in \mathbb{Z}^{n},\left\langle z_{j}, w_{i}\right\rangle \in \mathbb{Z}$. Furthermore, $t_{i} \in \mathbb{N},\left\langle v, z_{j}\right\rangle=$ $h_{K}(v) \in \mathbb{Z}$ and $w \in \mathbb{Z}^{n}$ implies that $\left\langle z_{j}, t_{i} v+w\right\rangle \in \mathbb{Z}$. Given this, we must have $\left\langle z_{j}, w_{i}\right\rangle=\left\langle z_{j}, t_{i} v+w\right\rangle \quad \forall j \in\{1, \ldots, k\}, i \geq 1$ and hence we get $\left\langle x, w_{i}\right\rangle=$ $\left\langle x, t_{i} v+w\right\rangle \quad \forall x \in S, i \geq 1$.

Let $w^{\prime}=w_{i}$ where $i=\max \left\{N_{1}, N_{2}\right\}$. Now examine the set $L=\left\{x:\left\langle x, w^{\prime}\right\rangle \leq\right.$ $\left.\left\lfloor h_{K}\left(w^{\prime}\right)\right\rfloor\right\} \cap S$. Here we get that $\left\langle x, w_{i}\right\rangle \leq t_{i} h_{K}(v)+\left\lfloor h_{F_{v}(K)}(w)\right\rfloor$ and $\langle x, v\rangle=$ $h_{K}(v)$ for all $x \in L$ Hence, we see that $\left\langle x, w_{i}-t_{i} v\right\rangle \leq\left\lfloor h_{F_{v}(K)}(w)\right\rfloor$ for all $x \in L$. Furthermore, since $\left\langle x, w_{i}-t_{i} v\right\rangle=\langle x, w\rangle$ for all $x \in L \subseteq S$, we have that $\langle x, w\rangle \leq\left\lfloor h_{F_{v}(K)}(w)\right\rfloor$ for all $x \in L$, as needed. 


\subsection{Separating All Points in $\boldsymbol{F}_{v} \backslash \operatorname{aff}_{I}\left(H_{v}^{=}\right)$}

Since the guarantees on the lifted CG cuts produced in the previous section are restricted to $\operatorname{aff}_{I}\left(H_{v}^{=}\right)$, we must still deal with the points in $F_{v} \backslash \operatorname{aff}_{I}\left(H_{v}^{=}\right)$. In this section, we show that points in $F_{v} \backslash \operatorname{aff}_{I}\left(H_{v}^{=}\right)$can be separated by using a finite number of CG cuts in Proposition 2. To prove this, we will need Kronecker's theorem on simultaneous diophantine approximation which is stated next. See Niven [14] or Cassels [3] for a proof.

Theorem 3. Let $\left(x_{1}, \ldots, x_{n}\right) \in \mathbb{R}^{n}$ be such that the numbers $x_{1}, \ldots, x_{n}, 1$ are linearly independent over $\mathbb{Q}$. Then the set $\left\{\left(n x_{1}(\bmod 1), \ldots, n x_{n}(\bmod 1)\right)\right.$ : $n \in \mathbb{N}\}$ is dense in $[0,1)^{n}$.

We state the following lemmas without proof which allow us to normalize vector $v$ defining $F_{v}$ and $H_{v}^{=}$and simplify the analysis that follows.

Lemma 2. Let $K \subseteq \mathbb{R}^{n}$ be a closed convex set, and let $T: \mathbb{R}^{n} \rightarrow \mathbb{R}^{n}$ be an invertible linear transformation. Then $h_{K}(v)=h_{T K}\left(T^{-t} v\right)$ and $F_{v}(K)=$ $T^{-1}\left(F_{T^{-t} v}(T K)\right)$ for all $v \in \mathbb{R}^{n}$. Furthermore, if $T$ is a unimodular transformation, then $C C(K)=T^{-1}(C C(T K))$.

Lemma 3. Take $v \in \mathbb{R}^{n}$. Then there exists an unimodular transformation $T$ : $\mathbb{R}^{n} \rightarrow \mathbb{R}^{n}$ and $\lambda \in \mathbb{Q}_{>0}$ such that for $v^{\prime}=\lambda T v$ we get that

$$
v^{\prime}=(\underbrace{0, \ldots, 0}_{t \text { times }}, \underbrace{1}_{s \text { times }}, \alpha_{1}, \ldots, \alpha_{r}),
$$

where $t, r \in \mathbb{Z}_{+}, s \in\{0,1\}$, and $\left\{1, \alpha_{1}, \ldots, \alpha_{r}\right\}$ are linearly independent over $\mathbb{Q}$. Furthermore, we have that $\mathcal{D}(v)=\inf \left\{\operatorname{dim}(W): v \in W, W=\left\{x \in \mathbb{R}^{n}: A x=\right.\right.$ $\left.0\}, A \in \mathbb{Q}^{m \times n}\right\}=s+r$.

We now show that the points in $F_{v} \backslash \operatorname{aff}_{I}\left(H_{v}^{=}\right)$can be separated using a finite number of CG cuts. We first give a rough sketch of the proof. We restrict to the case where $\operatorname{aff}_{I}\left(H_{v}^{=}\right) \neq \emptyset$. From here one can verify that any rational affine subspace contained in $\operatorname{aff}\left(H_{v}^{=}\right)$must also lie in $\operatorname{aff}_{I}\left(H_{v}^{=}\right)$. Next we use Kronecker's theorem to build a finite set $C \subseteq \mathbb{Z}^{n}$, where each vector in $C$ is at distance at most $\epsilon$ from some scaling of $v$, and where $v$ can be expressed as a non-negative combination of the vectors in $C$. By choosing $\epsilon$ and the scalings of $v$ appropriately, we can ensure that the $\mathrm{CG}$ cuts derived from $C$ dominate the inequality $\langle v, x\rangle \leq h_{K}(v)$, i.e. $C C(K, C) \subseteq H_{v}$. If $C C(K, C)$ lies in the interior of $H_{v}(K)$, we have separated all of $H_{v}^{=}$(including $F_{v} \backslash \operatorname{aff}_{I}\left(H_{v}^{=}\right)$) and hence are done. Otherwise, $T:=C C(K, C) \cap H_{v}^{=}$is a face of a rational polyhedron, and therefore $\operatorname{aff}(T)$ is a rational affine subspace. Since $\operatorname{aff}(T) \subseteq \operatorname{aff}\left(H_{v}^{=}\right)$, as discussed above $T \subseteq \operatorname{aff}(T) \subseteq \operatorname{aff}_{I}\left(H_{v}^{=}\right)$as required.

Proposition 2. Let $K \subseteq \mathbb{R}^{n}$ be a compact convex set and $v \in \mathbb{R}^{n}$. Then there exists $C \subseteq \mathbb{Z}^{n},|C| \leq \mathcal{D}(v)+1$, such that

$$
C C(K, C) \subseteq H_{v}(K) \quad \text { and } \quad C C(K, C) \cap H_{v}^{=}(K) \subseteq \operatorname{aff}_{I}\left(H_{v}^{=}(K)\right) .
$$


Proof. By scaling $v$ by a positive scalar if necessary, we may assume that $h_{K}(v) \in$ $\{0,1,-1\}$. Let $T$ and $\lambda$ denote the transformation and scaling promised for $v$ in Lemma 3. Note that $T^{-t}\left\{x \in \mathbb{R}^{n}:\langle v, x\rangle=h_{K}(v)\right\}=\left\{x \in \mathbb{R}^{n}:\left\langle v, T^{t} x\right\rangle=\right.$ $\left.h_{K}(v)\right\}=\left\{x \in \mathbb{R}^{n}:\langle\lambda T v, x\rangle=h_{T^{-t} K}(\lambda T v)\right\}$.

Now let $v^{\prime}=\lambda T v$ and $b^{\prime}=h_{T^{-t} K}(\lambda T v)$. By Lemma 2, it suffices to prove the statement for $v^{\prime}$ and $K^{\prime}=T^{-t} K$. Now $v^{\prime}$ has the form (5) where $t, r \in \mathbb{Z}_{+}$, $s \in\{0,1\}$, and $\left(1, \alpha_{1}, \ldots, \alpha_{r}\right)$ are linearly independent over $\mathbb{Q}$. For convenience, let $k=s+t$, where we note that $v_{k+1}^{\prime}, \ldots, v_{k+r}^{\prime}=\left(\alpha_{1}, \ldots, \alpha_{r}\right)$.

Claim 1: Let $S=\left\{x \in \mathbb{Z}^{n}:\left\langle v^{\prime}, x\right\rangle=b^{\prime}\right\}$. Then $S$ satisfies one of the following: (1) $S=\mathbb{Z}^{t} \times b^{\prime} \times 0^{r}: s=1, b^{\prime} \in \mathbb{Z},(2) S=\mathbb{Z}^{t} \times 0^{r}: s=0, b^{\prime}=0$, (3) $S=\emptyset$ : $s=0, b^{\prime} \neq 0$ or $s=1, b^{\prime} \notin \mathbb{Z}$.

Claim 2: Let $I=\left\{n v^{\prime}(\bmod 1): n \in N\right\}$. Then Theorem 3 implies that $I$ is dense in $0^{k} \times[0,1)^{r}$.

Due to space restriction, we skip the proofs of these two claims and from now on we only consider the case where $S \neq \emptyset$.

Claim 3: There exists $a_{1}, \ldots, a_{r+1} \subseteq \mathbb{Z}^{n}$ and $\lambda_{1}, \ldots, \lambda_{r+1} \geq 0$ such that $\sum_{i=1}^{r+1} \lambda_{i} a_{i}$ $=v^{\prime}$ and $\sum_{i=1}^{r+1} \lambda_{i}\left\lfloor h_{K}^{\prime}\left(a_{i}\right)\right\rfloor \leq b^{\prime}$.

Since $K^{\prime}$ is compact, there exists $R>0$ such that $K^{\prime} \subseteq R B^{n}$. Take the subspace $W=0^{k} \times \mathbb{R}^{r}$. Let $w_{1}, \ldots, w_{r+1} \in W \cap S^{n-1}$, be any vectors such that for some $0<\epsilon<1$ we have $\sup _{1 \leq i \leq r+1}\left\langle w_{i}, d\right\rangle \geq \epsilon$ for all $d \in S^{n-1} \cap W$ (e.g. $w_{1}, \ldots, w_{r+1}$ are the vertices of a scaled isotropic $r$-dimensional simplex). Let $a=\frac{1}{8} \min \left\{\frac{1}{R}, \epsilon\right\}$, and $b=\frac{1}{2} \epsilon a$. Now, for $1 \leq i \leq r+1$ define $E_{i}=\{x: x \in$ $\left.a w_{i}+b\left(B^{n} \cap W\right)(\bmod 1)\right\}$. Since $W=0^{k} \times \mathbb{R}^{r}$, note that $E_{i} \subseteq 0^{k} \times[0,1)^{r}$. By Claim 2 the set $I$ is dense in $0^{k} \times[0,1)^{r}$. Furthermore each set $E_{i}$ has non-empty interior with respect to the subspace topology on $0^{k} \times[0,1)^{r}$. Hence for all $i$, $1 \leq i \leq r+1$, we can find $n_{i} \in \mathbb{N}$ such that $n_{i} v^{\prime}(\bmod 1) \in E_{i}$.

Now $n_{i} v^{\prime}(\bmod 1) \in E_{i}$, implies that for some $\delta_{i}^{\prime} \in E_{i}, n_{i} v^{\prime}-\delta_{i}^{\prime} \in \mathbb{Z}^{n}$. Furthermore $\delta_{i}^{\prime} \in E_{i}$ implies that there exists $\delta_{i} \in a w_{i}+b\left(B^{n} \cap W\right)$ such that $\delta_{i}^{\prime}-\delta_{i} \in \mathbb{Z}^{n}$. Hence $\left(n_{i} v^{\prime}-\delta_{i}^{\prime}\right)+\left(\delta_{i}^{\prime}-\delta_{i}\right)=n_{i} v^{\prime}-\delta_{i} \in \mathbb{Z}^{n}$. Let $a_{i}=n_{i} v^{\prime}-\delta_{i}$. Note that $\left\|a_{i}-n_{i} v^{\prime}\right\|=\left\|-\delta_{i}\right\| \leq a+b \leq 2 a \leq 1 /(4 R)$. We claim that $\left\lfloor h_{K^{\prime}}\left(a_{i}\right)\right\rfloor \leq$ $h_{K^{\prime}}\left(n_{i} v^{\prime}\right)$. First note that $h_{K^{\prime}}\left(n_{i} v^{\prime}\right)=n_{i} b^{\prime}$. Since we assume that $S \neq \emptyset$, we must have that $b^{\prime} \in \mathbb{Z}$ and hence $n_{i} b^{\prime} \in \mathbb{Z}$. Now note that

$$
\begin{aligned}
h_{K^{\prime}}\left(a_{i}\right) & =h_{K^{\prime}}\left(\left(a_{i}-n_{i} v^{\prime}\right)+n_{i} v^{\prime}\right) \leq h_{K^{\prime}}\left(n_{i} v^{\prime}\right)+h_{K^{\prime}}\left(a_{i}-n_{i} v^{\prime}\right) \\
& =n_{i} b^{\prime}+h_{K^{\prime}}\left(-\delta_{i}\right) \\
& \leq n_{i} b^{\prime}+h_{R B^{n}}\left(-\delta_{i}\right) \leq n_{i} b^{\prime}+R\left\|\delta_{i}\right\| \leq n_{i} b^{\prime}+R\left(\frac{1}{4 R}\right)=n_{i} b^{\prime}+\frac{1}{4} .
\end{aligned}
$$

Therefore we have that $\left\lfloor h_{K^{\prime}}\left(a_{i}\right)\right\rfloor \leq\left\lfloor n_{i} b^{\prime}+\frac{1}{4}\right\rfloor=n_{i} b^{\prime}=h_{K^{\prime}}\left(n_{i} v^{\prime}\right)$, since $n_{i} b^{\prime} \in \mathbb{Z}$.

We claim that $\frac{a \epsilon}{4} B^{n} \cap W \subseteq \operatorname{conv}\left\{\delta_{1}, \ldots, \delta_{r+1}\right\}$. First note that by construction, $\operatorname{conv}\left\{\delta_{1}, \ldots, \delta_{r+1}\right\} \subseteq \bar{W}$. Hence if the conclusion is false, then by the separator theorem there exists $d \in W \cap S^{n-1}$ such that $h_{\frac{a \epsilon}{4} B^{n} \cap W}(d)=\frac{a \epsilon}{4}>$ 
$\sup _{1 \leq i \leq r+1}\left\langle d, \delta_{i}\right\rangle$. For each $i, 1 \leq i \leq r+1$, we write $\delta_{i}=a w_{i}+b z_{i}$ where $\left\|z_{i}\right\| \leq 1$. Now note that

$$
\begin{aligned}
\sup _{1 \leq i \leq r+1}\left\langle d, \delta_{i}\right\rangle & =\sup _{1 \leq i \leq r+1}\left\langle d, a w_{i}+b z_{i}\right\rangle=\sup _{1 \leq i \leq r+1} a\left\langle d, w_{i}\right\rangle+b\left\langle d, z_{i}\right\rangle \\
& \geq \sup _{1 \leq i \leq r+1} a\left\langle d, w_{i}\right\rangle-b\|d\|\left\|z_{i}\right\| \geq a \epsilon-b=\frac{a \epsilon}{2}>\frac{a \epsilon}{4}
\end{aligned}
$$

a contradiction. Hence there exists $\lambda_{1}, \ldots, \lambda_{r+1} \geq 0$ and $\sum_{i=1}^{r+1} \lambda_{i} n_{i}=1$ such that $\sum_{i=1}^{r+1} \lambda_{i} \delta_{i}=0$.

Now we see that

$$
\sum_{i=1}^{r+1} \lambda_{i} a_{i}=\sum_{i=1}^{r+1} \lambda_{i} n_{i} v^{\prime}+\sum_{i=1}^{r+1} \lambda_{i}\left(a_{i}-n_{i} v^{\prime}\right)=\left(\sum_{i=1}^{r+1} \lambda_{i} n_{i}\right) v^{\prime}-\sum_{i=1}^{r+1} \lambda_{i} \delta_{i}=\left(\sum_{i=1}^{r+1} \lambda_{i} n_{i}\right) v^{\prime} .
$$

Next note that

$$
\sum_{i=1}^{r+1} \lambda_{i}\left\lfloor h_{K^{\prime}}\left(a_{i}\right)\right\rfloor \leq \sum_{i=1}^{r+1} \lambda_{i} h_{K^{\prime}}\left(n_{i} v^{\prime}\right)=h_{K^{\prime}}\left(\left(\sum_{i=1}^{r+1} \lambda_{i} n_{i}\right) v^{\prime}\right) .
$$

Claim 4: Let $C=\left\{a_{i}\right\}_{i=1}^{r+1}$ for the $a_{i}$ 's from Claim 3. Then $C C(K, C) \cap\{x$ : $\left.\left\langle v^{\prime}, x\right\rangle=b^{\prime}\right\} \subseteq \operatorname{aff}(S)$.

Examine the set $P=\left\{x:\left\langle v^{\prime}, x\right\rangle=b^{\prime},\left\langle a_{i}, x\right\rangle \leq\left\lfloor h_{K^{\prime}}\left(a_{i}\right)\right\rfloor, 1 \leq i \leq l+1\right\}$. From the proof of Claim 3, we know that for each $i, 1 \leq i \leq r+1$, we have $\left\lfloor h_{K^{\prime}}\left(a_{i}\right)\right\rfloor \leq h_{K^{\prime}}\left(n_{i} v^{\prime}\right)=n_{i} b^{\prime}$ and hence $\left\langle n_{i} v^{\prime}-a_{i}, x\right\rangle=\left\langle\delta_{i}, x\right\rangle \geq 0$, is a valid inequality for $P$. Now, from the proof of Claim 3 , we have

$$
\frac{a \epsilon}{4} B^{n} \cap W \subseteq \operatorname{conv}\left\{\delta_{1}, \ldots, \delta_{r+1}\right\} .
$$

We claim that for all $H \subseteq\{1, \ldots, r+1\},|H|=r$, the set $\left\{\delta_{i}: i \in H\right\}$ is linearly independent. Assume not, then WLOG we may assume that $\delta_{1}, \ldots, \delta_{r}$ are not linearly independent. Hence there exists $d \in S^{n-1} \cap W$, such that $\left\langle d, \delta_{i}\right\rangle=0$ for all $1 \leq i \leq n$. Now by possibly switching $d$ to $-d$, we may assume that $\left\langle d, \delta_{r+1}\right\rangle \leq 0$. Hence we get that $\sup _{1 \leq i \leq r+1}\left\langle d, \delta_{i}\right\rangle \leq 0$ in contradiction to (8).

Now let $\lambda_{1}, \ldots, \lambda_{r+1} \geq 0, \sum_{i=1}^{r+1} \lambda_{i} n_{i}=1$ be a combination such that $\sum_{i=1}^{r+1} \lambda_{i} \delta_{i}=0$. Note that $\lambda_{1}, \ldots, \lambda_{r+1}$ forms a linear dependency on $\delta_{1}, \ldots, \delta_{r+1}$, and hence by the previous claim we must have that $\lambda_{i}>0$ for all $1 \leq i \leq r+1$.

We claim for $P \subseteq W^{\perp}$. To see this, note that $0=\langle x, 0\rangle=\left\langle x, \sum_{i=1}^{r+1} \lambda_{i} \delta_{i}\right\rangle=$ $\sum_{i=1}^{r+1} \lambda_{i}\left\langle x, \delta_{i}\right\rangle$ for every $x \in P$. Now since $\operatorname{span}\left(\delta_{1}, \ldots, \delta_{r+1}\right)=W$, we see that $\left\langle x, \delta_{i}\right\rangle=0$ for all $1 \leq i \leq r+1$ iff $x \in W^{\perp}$. Hence if $x \notin W^{\perp}$, then by the above equation and the fact that $\lambda_{i}>0$ for all $i \in\{1, \ldots, r+1\}$, there exists $i, j \in\{1, \ldots, r+1\}$ such that $\left\langle x, \delta_{i}\right\rangle>0$ and $\left\langle x, \delta_{j}\right\rangle<0$. But then $x \notin P$, since $\left\langle x, \delta_{j}\right\rangle<0$, a contradiction. Now $W=0^{k} \times \mathbb{R}^{r}$, hence $W^{\perp}=\mathbb{R}^{k} \times 0^{r}$. To complete the proof we see that $P \subseteq\left\{x: x \in \mathbb{R}^{k} \times 0^{r},\left\langle v^{\prime}, x\right\rangle=b^{\prime}\right\}=\operatorname{aff}(S)$. 


\subsection{Lifting the CG Closure of an Exposed Face of $K$}

Proposition 3. Let $K \subseteq \mathbb{R}^{n}$ be a compact convex set. Take $v \in \mathbb{R}^{n}$. Assume that $C C\left(F_{v}(K)\right)$ is finitely generated. Then $\exists S \subseteq \mathbb{Z}^{n},|S|<\infty$, such that $C C(K, S)$ is a polytope and

$$
\begin{gathered}
C C(K, S) \cap H_{v}^{=}(K)=C C\left(F_{v}(K)\right) \\
C C(K, S) \subseteq H_{v} .
\end{gathered}
$$

Proof. The right to left containment in (9) is direct from $C C\left(F_{v}(K)\right) \subseteq C C(K, S)$ as every CG cut for $K$ is a CG cut for $F_{v}(K)$. For the reverse containment and for (10) we proceed as follows.

Using Proposition 2 there exists $S_{1} \subseteq \mathbb{Z}^{n}$ such that $C C\left(K, S_{1}\right) \cap H_{v}^{=}(K) \subseteq$ $\operatorname{aff}_{I}\left(H_{v}^{=}(K)\right)$ and $C C\left(K, S_{1}\right) \subseteq\left\{x \in \mathbb{R}^{n}:\langle v, x\rangle \leq h_{K}(v)\right\}$. Next let $G \subseteq \mathbb{Z}^{n}$ be such that $C C\left(F_{v}(K), G\right)=C C\left(F_{v}(K)\right)$. For each $w \in G$, by Proposition 1 there exists $w^{\prime} \in \mathbb{Z}^{n}$ such that $C C\left(K, w^{\prime}\right) \cap \operatorname{aff}_{I}\left(H_{v}^{=}(K)\right) \subseteq C C\left(F_{v}(K), w\right) \cap$ $\operatorname{aff}_{I}\left(H_{v}^{=}(K)\right)$. For each $w \in G$, add $w^{\prime}$ above to $S_{2}$. Now note that

$$
\begin{aligned}
C C\left(K, S_{1} \cup S_{2}\right) \cap H_{v}^{=}(K) & =C C\left(K, S_{1}\right) \cap C C\left(K, S_{2}\right) \cap H_{v}^{=}(K) \\
& \subseteq C C\left(K, S_{2}\right) \cap \operatorname{aff}_{I}\left(H_{v}^{=}(K)\right) \\
& =C C\left(F_{v}(K), G\right) \cap \operatorname{aff}\left(H_{v}^{=}(K)\right) \subseteq C C\left(F_{v}(K)\right) .
\end{aligned}
$$

Now let $S_{3}=\left\{ \pm e_{i}: 1 \leq i \leq n\right\}$. Note that since $K$ is compact $C C\left(K, S_{3}\right)$ is a cuboid with bounded side lengths, and hence is a polytope. Letting $S=$ $S_{1} \cup S_{2} \cup S_{3}$, yields the desired result.

We now obtain a generalization of the classical result known for rational polyhedra.

Corollary 1. Let $K$ be a compact convex set and let $F$ be an exposed face of $K$, then we have that $C C(F)=C C(K) \cap F$.

\section{Approximation of the CG Closure}

\subsection{Approximation 1 of the CG Closure}

In this section, we construct a first approximation of the CG closure of $K$. Under the assumption that the CG closure of every proper exposed face is finitely generated, we use a compactness argument to construct a finite set of CG cuts $S \subseteq \mathbb{Z}^{n}$ such that $C C(K, S) \subseteq K \cap \operatorname{aff}_{I}(K)$. We use the following lemma (stated without proof) to simplify the analysis of integral affine subspaces.

Lemma 4. Take $A \in \mathbb{R}^{m \times n}$ and $b \in \mathbb{R}^{m}$. Then there exists $\lambda \in \mathbb{R}^{m}$ such that for $a^{\prime}=\lambda A, b^{\prime}=\lambda b$, we have that $\left\{x \in \mathbb{Z}^{n}: A x=b\right\}=\left\{x \in \mathbb{Z}^{n}: a^{\prime} x=b^{\prime}\right\}$.

Proposition 4. Let $\emptyset \neq K \subseteq \mathbb{R}^{n}$ be a compact convex set. If $C C\left(F_{v}(K)\right)$ is finitely generated for any proper exposed face $F_{v}(K)$ then $\exists S \subseteq \mathbb{Z}^{n},|S|<\infty$, such that $C C(K, S) \subseteq K \cap \operatorname{aff}_{I}(K)$ and $C C(K, S)$ is a polytope. 
Proof. Let us express aff $(K)$ as $\left\{x \in \mathbb{R}^{n}: A x=b\right\}$. Note that aff $(K) \neq \emptyset$ since $K \neq \emptyset$. By Lemma 4 there exists $\lambda, c=\lambda A$ and $d=\lambda b$, and such that $\operatorname{aff}(K) \cap \mathbb{Z}^{n}=\left\{x \in \mathbb{Z}^{n}:\langle c, x\rangle=b\right\}$. Since $h_{K}(c)=b$ and $h_{K}(-c)=-b$, using Proposition 2 on $c$ and $-c$, we can find $S_{A} \subseteq \mathbb{Z}^{n}$ such that $C C\left(K, S_{A}\right) \subseteq$ aff $(\{x \in$ $\left.\left.\mathbb{Z}^{n}:\langle c, x\rangle=b\right\}\right)=\operatorname{aff}_{I}(K)$.

Express aff $(K)$ as $W+a$, where $W \subseteq \mathbb{R}^{n}$ is a linear subspace and $a \in \mathbb{R}^{n}$. Now take $v \in W \cap S^{n-1}$. Note that $F_{v}(K)$ is a proper exposed face and hence, by assumption, $C C\left(F_{v}(K)\right)$ is finitely generated. Hence by Proposition 3 there exists $S_{v} \subseteq \mathbb{Z}^{n}$ such that $C C\left(K, S_{v}\right)$ is a polytope, $C C\left(K, S_{v}\right) \cap H_{v}^{=}(K)=$ $C C\left(F_{v}(K)\right)$ and $C C\left(K, S_{v}\right) \subseteq\left\{x \in \mathbb{R}^{n}:\langle x, v\rangle \leq h_{K}(v)\right\}$. Let $K_{v}=C C\left(K, S_{v}\right)$, then we have the following claim whose proof we skip because of lack of space. Claim: $\exists$ open neighborhood $N_{v}$ of $v$ in $W \cap S^{n-1}$ such that $v^{\prime} \in N_{v} \Rightarrow$ $h_{K_{v}}\left(v^{\prime}\right) \leq h_{K}\left(v^{\prime}\right)$.

Note that $\left\{N_{v}: v \in W \cap S^{n-1}\right\}$ forms an open cover of $W \cap S^{n-1}$, and since $W \cap S^{n-1}$ is compact, there exists a finite subcover $N_{v_{1}}, \ldots, N_{v_{k}}$ such that $\bigcup_{i=1}^{k} N_{v_{i}}=W \cap S^{n-1}$. Now let $S=S_{A} \cup \cup_{i=1}^{k} S_{v_{i}}$. We claim that $C C(K, S) \subseteq K$. Assume not, then there exists $x \in C C(K, S) \backslash K$. Since $C C(K, S) \subseteq C C\left(K, S_{A}\right) \subseteq$ $W+a$ and $K \subseteq W+a$, by the separator theorem there exists $w \in W \cap S^{n-1}$ such that $h_{K}(w)=\sup _{y \in K}\langle y, w\rangle<\langle x, w\rangle \leq h_{C C(K, S)}(w)$. Since $w \in W \cap S^{n-1}$, there exists $i, 1 \leq i \leq k$, such that $w \in N_{v_{i}}$. Note then we obtain that $h_{C C(K, S)}(w) \leq h_{C C\left(K, S_{v_{i}}\right)}(w)=h_{K_{v_{i}}}(w) \leq h_{K}(w)$, a contradiction. Hence $C C(K, S) \subseteq K$ as claimed. $C C(K, S)$ is a polytope because it is the intersection of polyhedra of which at least one is a polytope.

\subsection{Approximation 2 of the CG Closure}

In this section, we augment the first approximation of the $C C(K)$ with a finite number of extra $\mathrm{CG}$ cuts so that this second approximation matches $C C(K)$ on the relative boundary of $K$.

To achieve this, we observe that our first approximation of $C C(K)$ is polyhedral and contained in $K$, and hence its intersection with the relative boundary of $K$ is contained in the union of a finite number of proper exposed faces of $K$. Therefore, by applying Proposition 3 to each such face (i.e. adding their lifted CG closure), we can match $C C(K)$ on the relative boundary as required. The following lemma (stated without proof) makes precise the previous statements.

Lemma 5. Let $K \subseteq \mathbb{R}^{n}$ be a convex set and $P \subseteq K$ be a polytope. Then there exists $F_{v_{1}}, \ldots, F_{v_{k}} \subseteq K$, proper exposed faces of $K$, such that $P \cap \operatorname{relbd}(K) \subseteq \bigcup_{i=1}^{k}$ $F_{v_{i}}$

Proposition 5. Let $K \subseteq \mathbb{R}^{n}$ be a compact convex set. If $C C\left(F_{v}\right)$ is finitely generated for any proper exposed face $F_{v}$ then $\exists S \subseteq \mathbb{Z}^{n},|S|<\infty$, such that

$$
\begin{aligned}
C C(K, S) & \subseteq K \cap \operatorname{aff}_{I}(K) \\
C C(K, S) \cap \operatorname{relbd}(K) & =C C(K) \cap \operatorname{relbd}(K)
\end{aligned}
$$


Proof. By Proposition 4, there exists $S_{I} \subseteq \mathbb{Z}^{n},\left|S_{I}\right|<\infty$, such that $C C\left(K, S_{I}\right) \subseteq$ $K \cap \operatorname{aff}_{I}(K)$ and $C C\left(K, S_{I}\right)$ is a polytope. Since $C C\left(K, S_{I}\right) \subseteq K$ is a polytope, let $F_{v_{1}}, \ldots, F_{v_{k}}$ be the proper exposed faces of $K$ given by Lemma 5 . By Proposition 3 , there exists $S_{i} \subseteq \mathbb{Z}^{n},\left|S_{i}\right|<\infty$, such that $C C\left(K, S_{i}\right) \cap H_{v_{i}}=C C\left(F_{v_{i}}\right)$. Let $S=S_{I} \cup \cup_{i=1}^{k} S_{i}$. We claim that $C C(K, S) \cap \operatorname{relbd}(K) \subseteq C C(K) \cap \operatorname{relbd}(K)$. For this note that $x \in C C(K, S) \cap \operatorname{relbd}(K)$ implies $x \in C C\left(K, S_{I}\right) \cap \operatorname{relbd}(K)$, and hence there exists $i, 1 \leq i \leq k$, such that $x \in F_{v_{i}}$. Then $x \in C C(K, S) \cap H_{v_{i}} \subseteq$ $C C\left(K, S_{i}\right) \cap H_{v_{i}}=C C\left(F_{v_{i}}\right) \subseteq C C(K) \cap \operatorname{relbd}(K)$. The reverse inclusion is direct.

\section{Proof of Theorem}

Finally, we have all the ingredients to prove the main result of this paper. The proof is by induction on the dimension of $K$. Trivially, the result holds for zero dimensional convex bodies. Now using the induction hypothesis, we can construct the second approximation of $C C(K)$ using Proposition 5 (since it assumes that the CG closure of every exposed face is finitely generated). Lastly, we observe that any $\mathrm{CG}$ cut for $K$ not dominated by those already considered in the second approximation of $C C(K)$ must separate a vertex of this approximation lying in the relative interior of $K$. From here, it is not difficult to show that only a finite number of such cuts exists, thereby proving the polyhedrality of $C C(K)$. The proof here is similar to the one used for strictly convex sets, with the additional technicality that here $\operatorname{aff}(K)$ may be irrational.

Theorem 4. Let $K \subseteq \mathbb{R}^{n}$ be a non-empty compact convex set. Then $C C(K)$ is finitely generated.

Proof. We proceed by induction on the affine dimension of $K$. For the base case, $\operatorname{dim}(\operatorname{aff}(K))=0$, i.e. $K=\{x\}$ is a single point. Here it is easy to see that setting $S=\left\{ \pm e_{i}: i \in\{1, \ldots, n\}\right\}$, we get that $C C(K, S)=C C(K)$. The base case thus holds.

Now for the inductive step let $0 \leq k<n$ let $K$ be a compact convex set where $\operatorname{dim}(\operatorname{aff}(K))=k+1$ and assume the result holds for sets of lower dimension. By the induction hypothesis, we know that $C C\left(F_{v}\right)$ is finitely generated for every proper exposed face $F_{v}$ of $K$, since $\operatorname{dim}\left(F_{v}\right) \leq k$. By Proposition 5 , there exists a set $S \subseteq \mathbb{Z}^{n},|S|<\infty$, such that (11) and (12) hold. If $C C(K, S)=\emptyset$, then we are done. So assume that $C C(K, S) \neq \emptyset$. Let $A=\operatorname{aff}_{I}(K)$. Since $C C(K, S) \neq \emptyset$, we have that $A \neq \emptyset$ (by (11)), and so we may pick $t \in A \cap \mathbb{Z}^{n}$. Note that $A-t=W$, where $W$ is a linear subspace of $\mathbb{R}^{n}$ satisfying $W=\operatorname{span}\left(W \cap \mathbb{Z}^{n}\right)$. Let $L=W \cap \mathbb{Z}^{n}$. Since $t \in \mathbb{Z}^{n}$, we easily see that $C C(K-t, T)=C C(K, T)-t$ for all $T \subseteq \mathbb{Z}^{n}$. Therefore $C C(K)$ is finitely generated iff $C C(K-t)$ is. Hence replacing $K$ by $K-t$, we may assume that $\operatorname{aff}_{I}(K)=W$.

Let $\pi_{W}$ denote the orthogonal projection onto $W$. Note that for all $x \in W$, and $z \in \mathbb{Z}^{n}$, we have that $\langle z, x\rangle=\left\langle\pi_{W}(z), x\right\rangle$. Now since $C C(K, S) \subseteq K \cap W$, we see that for all $z \in \mathbb{Z}^{n}, C C(K, S \cup\{z\})=C C(K, S) \cap\left\{x:\langle z, x\rangle \leq\left\lfloor h_{K}(z)\right\rfloor\right\}=$ $C C(K, S) \cap\left\{x:\left\langle\pi_{W}(z), x\right\rangle \leq\left\lfloor h_{K}(z)\right\rfloor\right\}$. Let $L^{*}=\pi_{W}\left(\mathbb{Z}^{n}\right)$. Since $W$ is a rational 
subspace, we have that $L^{*}$ is full dimensional lattice in $W$. Now fix an element of $w \in L^{*}$ and examine $V_{w}:=\left\{\left\lfloor h_{K}(z)\right\rfloor: \pi_{W}(z)=w, z \in \mathbb{Z}^{n}\right\}$. Note that $V_{w} \subseteq \mathbb{Z}$. We claim that $\inf \left(V_{w}\right) \geq-\infty$. To see this, note that

$$
\begin{aligned}
\inf \left\{\left\lfloor h_{K}(z)\right\rfloor: \pi_{W}(z)=w, z \in \mathbb{Z}^{n}\right\} & \geq \inf \left\{\left\lfloor h_{K \cap W}(z)\right\rfloor: \pi_{W}(z)=w, z \in \mathbb{Z}^{n}\right\} \\
& =\inf \left\{\left\lfloor h_{K \cap W}\left(\pi_{W}(z)\right)\right\rfloor: \pi_{W}(z)=w, z \in \mathbb{Z}^{n}\right\} \\
& =\left\lfloor h_{K \cap W}(w)\right\rfloor>-\infty .
\end{aligned}
$$

Now since $V_{w}$ is a lower bounded set of integers, there exists $z_{w} \in \pi_{W}^{-1}(w) \cap$ $\mathbb{Z}^{n}$ such that $\inf \left(V_{w}\right)=\left\lfloor h_{K}\left(z_{w}\right)\right\rfloor$. From the above reasoning, we see that $C C\left(K, S \cup \pi_{W}^{-1}(z) \cap \mathbb{Z}^{n}\right)=C C\left(K, S \cup\left\{z_{w}\right\}\right)$. Now examine the set $C=\{w$ : $\left.w \in L^{*}, C C\left(K, S \cup\left\{z_{w}\right\}\right) \subsetneq C C(K, S)\right\}$. Here we get that

$C C(K)=C C\left(K, S \cup \mathbb{Z}^{n}\right)=C C\left(K, S \cup\left\{z_{w}: w \in L^{*}\right\}\right)=C C\left(K, S \cup\left\{z_{w}: w \in C\right\}\right)$.

From the above equation, if we show that $|C|<\infty$, then $C C(K)$ is finitely generated. To do this, we will show that there exists $R>0$, such that $C \subseteq R B^{n}$, and hence $C \subseteq L^{*} \cap R B^{n}$. Since $L^{*}$ is a lattice, $\left|L^{*} \cap R B^{n}\right|<\infty$ for any fixed $R$, and so we are done.

Now let $P=C C(K, S)$. Since $P$ is a polytope, we have that $P=\operatorname{conv}(\operatorname{ext}(P))$. Let $I=\operatorname{ext}(P) \cap \operatorname{relint}(K)$, and let $B=\operatorname{ext}(P) \cap \operatorname{relbd}(K)$. Hence $\operatorname{ext}(P)=$ $I \cup B$. By assumption on $C C(K, S)$, we know that for all $v \in B$, we have that $v \in C C(K)$. Hence for all $z \in \mathbb{Z}^{n}$, we must have that $\langle z, v\rangle \leq\left\lfloor h_{K}(z)\right\rfloor$ for all $v \in B$. Now assume that for some $z \in \mathbb{Z}^{n}, C C(K, S \cup\{z\}) \subsetneq C C(K, S)=P$. We claim that $\langle z, v\rangle>\left\lfloor h_{K}(z)\right\rfloor$ for some $v \in I$. If not, then $\langle v, z\rangle \leq\left\lfloor h_{K}(z)\right\rfloor$ for all $v \in \operatorname{ext}(P)$, and hence $C C(K, S \cup\{z\})=C C(K, S)$, a contradiction. Hence such a $v \in I$ must exist.

For $z \in \mathbb{Z}^{n}$, note that $h_{K}(z) \geq h_{K \cap W}(z)=h_{K \cap W}\left(\pi_{W}(z)\right)$. Hence $\langle z, v\rangle>$ $\left\lfloor h_{K}(z)\right\rfloor$ for $v \in I$ only if $\left\langle\pi_{W}(z), v\right\rangle=\langle z, v\rangle>\left\lfloor h_{K \cap W}\left(\pi_{W}(z)\right)\right\rfloor$. Let $C^{\prime}:=\{w \in$ $\left.L^{*}: \exists v \in I,\langle v, w\rangle>\left\lfloor h_{K \cap W}\right\rfloor(w)\right\}$. From the previous discussion, we see that $C \subseteq C^{\prime}$.

Since $I \subseteq \operatorname{relint}(K) \cap W=\operatorname{relint}(K \cap W)$ we have $\delta_{v}=\sup \left\{r \geq 0: r B^{n} \cap\right.$ $W+v \subseteq K \cap W\}>0$ for all $v \in I$. Let $\delta=\inf _{v \in I} \delta_{v}$. Since $|I|<\infty$, we see that $\delta>0$. Now let $R=\frac{1}{\delta}$. Take $w \in L^{*},\|w\| \geq R$. Note that $\forall v \in I$,

$\left\lfloor h_{K \cap W}(w)\right\rfloor \geq h_{K \cap W}(w)-1 \geq h_{\left(v+\delta B^{n}\right) \cap W}(w)-1=\langle v, w\rangle+\delta\|w\|-1 \geq\langle v, w\rangle$.

Hence $w \notin C^{\prime}$. Therefore $C \subseteq C^{\prime} \subseteq R B^{n}$ and $C C(K)$ is finitely generated.

\section{Remarks}

Using techniques developed in Proposition 2 and Lemma 4 it is possible to prove the following.

Theorem 5. Let $T=\left\{x \in \mathbb{R}^{n}: A x=b\right\}, A \in \mathbb{R}^{m \times n}, b \in \mathbb{R}^{m}$. The following holds: 
1. If $\operatorname{aff}_{I}(T)=\emptyset$, then for all $D>0$ there exists $z \in \mathbb{Z}^{n}$ such that $C C(T \cap$ $\left.D B^{n},\{z,-z\}\right)=\emptyset$.

2. If $\operatorname{aff}_{I}(T) \neq \emptyset$, then for all $D>0$ there exists $S \subseteq \mathbb{Z}^{n},|S|=n-\operatorname{dim}\left(\operatorname{aff}_{I}(T)\right)+$ 1 such that $C C\left(T \cap D B^{n}, S\right)=\operatorname{aff}_{I}(T)$.

The above result can be considered as a generalization of Integer Farkas' Lemma: If $A$ and $b$ are rational and $\operatorname{aff}_{I}(T)=\emptyset$, then it can be shown (we skip details due to lack of space) if $D>0$ is sufficiently large, then $C C\left(T \cap D B^{n},\{z,-z\}\right)=\emptyset$ implies that $C C(T,\{z,-z\})=\emptyset$ which is one half of regular Integer Farkas' Lemma.

\section{References}

1. Ben-Tal, A., Nemirovski, A.: Lectures on modern convex optimization: analysis, algorithms, and engineering applications. Society for Industrial and Applied Mathematics, Philadelphia, PA (2001)

2. Bonami, P., Dash, G.C.S., Fischetti, M., Lodi, A.: Projected Chvatal-Gomory Cuts for Mixed Integer Linear Programs. Mathematical Programming 113, 241-257 (2008)

3. Cassels, J.W.S.: An introduction to Diophantine approximation. Hafner, New York (1972)

4. Çezik, M.T., Iyengar, G.: Cuts for mixed 0-1 conic programming. Mathematical Programming 104, 179-202 (2005)

5. Chvátal, V.: Edmonds polytopes and a hierarchy of combinatorial problems. Discrete Mathematics 4, 305-337 (1973)

6. Dadush, D., Dey, S.S., Vielma, J.P.: The Chvátal-Gomory Closure of Strictly Convex Body (2010), to appear in Mathematics of Operations Research

7. Dey, S.S., Vielma, J.P.: The Chvátal-Gomory Closure of an Ellipsoid Is a Polyhedron. In: Eisenbrand, F., Shepherd, F.B. (eds.) IPCO XIV. Lecture Notes in Computer Science, vol. 6080, pp. 327-340. Springer (2010)

8. Dunkel, J., Schulz, A.S.: The Gomory-chvátal closure of a non-rational polytope is a rational polytope (2010), http://www.optimization-online.org/DB_HTML/ 2010/11/2803.html

9. Edmonds, J.: Paths, trees, and flowers. Canadian Journal of mathematics 17, 449467 (1965)

10. Fischetti, M., Lodi, A.: Optimizing over the first Chvátal closure. Mathematical Programming, Series B 110, 3-20 (2007)

11. Gomory, R.E.: Outline of an algorithm for integer solutions to linear programs. Bulletin of the American Mathematical Society 64, 275-278 (1958)

12. Grötschel, M., Padberg, M.: On the symmetric travelling salesman problem I: Inequalities. Math. Programming 16, 265-280 (1979)

13. Grötschel, M., Padberg, M.: On the symmetric travelling salesman problem II: Lifting theorems and facets. Math. Programming 16, 281-302 (1979)

14. Niven, I.M.: Diophantine approximations. Interscience Publishers, New York (1963)

15. Schrijver, A.: On cutting planes. Annals of Discrete Mathematics 9, 291-296 (1980), combinatorics 79 (Proc. Colloq., Univ. Montréal, Montreal, Que., 1979), Part II 